\title{
Research and Design of Car Reversing System
}

\author{
Zhiqiang $\mathrm{Xu}^{\mathrm{a}}$ \\ Guangdong University of Science \& Technology, Dongguan, 523083, China \\ a450521404@qq.com
}

Keywords: Car; Reversing; Radar system.

\begin{abstract}
Nowadays, with the improvement of people's living standards, automobiles have become the preferred mode of transportation for people. According to statistics, the number of automobiles in China and even the world each year has grown substantially, which has greatly improved people, s living standards. Promote the development of the automotive industry. On the other hand, the resulting traffic accidents have become more and more frequent. In particular, the loss of life and property caused by the lack of visibility during reversing the car is even more serious. Therefore, this article has designed a reversing radar system based on single-chip AT89C51, elaborated the structure of the system, hardware circuit design and software design. The system is simple in structure, extremely reliable and practical.
\end{abstract}

\section{Introduction}

Reversing radar, also known as parking assistance system, or computer reversing warning system, can effectively avoid obstacles or people that may be encountered when reversing, thus effectively avoiding economic losses and improving personal safety. Fast and accurate measurement of the distance between the obstacle and the car is a prerequisite for safely avoiding obstacles. Design and manufacture a simple car radar system, which needs to be launched through the keyboard control system, followed by a digital tube to display the distance of obstacles from the car, and again according to the display distance, the system self-detection, if the distance is less than the specified value, the system automatically alarm If the distance is greater than the specified value, the system will not alarm and operate normally. This subject takes SCM as the core and uses ultrasonic noncontact distance measurement technology to design automotive reversing radar system.

\section{Car Reversing System Composition}

The car reversing system mainly consists of an ultrasonic generator, an ultrasonic receiver, a SCM system, a digital tube display, and an alarm circuit.

\subsection{Ultrasonic Generator}

The ultrasonic generator is a device that converts mains into the corresponding high-frequency alternating current of the transducer to drive the transducer. It is an important part of the high-power ultrasonic system. It can also be called an electronic box. Ultrasonic drive power, ultrasonic controller. Ultrasonic generator adopts the world's leading helicial oscillating circuit structure, which increases the output power by more than $10 \%$ compared with the self-excited oscillating circuit structure. The ultrasonic amplifying circuit uses a linear amplifying circuit and a switching power supply circuit.

The ultrasonic generator generates a signal of a specific frequency. This signal can be a sine signal or a pulse signal. The specific frequency is the frequency at which the transducer operates. Ultrasonic equipment generally uses ultrasound frequencies of $20 \mathrm{KHz}, 25 \mathrm{KHz}, 28 \mathrm{KHz}, 33 \mathrm{KHz}, 40 \mathrm{KHz}, 60$ $\mathrm{KHz}, 80 \mathrm{KHz}, 100 \mathrm{KHz}$, or more, but it has not been widely used. Figure 1 below shows the internal structure of the ultrasonic generator. 


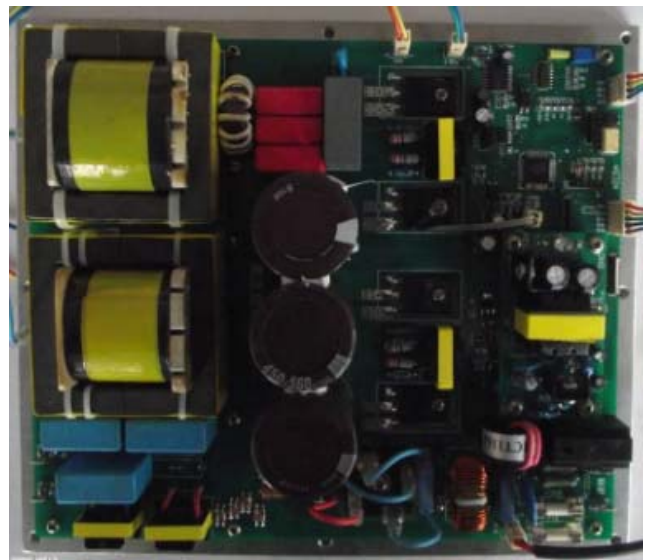

Figure 1. The internal structure of the ultrasonic generator

\subsection{Ultrasound Receiver}

The ultrasonic transmitter emits ultrasonic waves in a certain direction and starts timing at the same time as the transmission time. The ultrasonic wave propagates in the air, and immediately returns to the obstacle when the obstacle is encountered. The ultrasonic receiver stops the timing immediately after receiving the reflected wave. The propagation speed of the ultrasonic wave in air is $340 \mathrm{~m} / \mathrm{s}$. According to the time $\mathrm{t}$ recorded by the timer, the distance ( $\mathrm{s}$ ) from the obstacle to the obstacle can be calculated, that is: $\mathrm{s}=340 \mathrm{t} / 2$. The system is characterized by the use of SCM to control the ultrasonic emission and the time from the ultrasonic self-emission to the receiving and returning time, and is designed for the design of the car reversing system.

\subsection{Microcontroller System}

The single-chip microcomputer system refers to the external components necessary for the normal operation of the single-chip microcomputer and is mainly composed of a single-chip microcomputer, a crystal oscillator circuit, and a reset circuit. The input and output parts are implemented through the $\mathrm{I} / \mathrm{O}$ port of the microcontroller. In general, a single-chip microcomputer application system refers to an application system that is composed of a single-chip microcomputer, a peripheral interface circuit, and suitable software to achieve a specific function. In simple terms, a single-chip microcomputer is a microcontroller that integrates a central processing unit (CPU), a random access memory (RAM), a read-only memory timer chip, and some input/output interface circuits on one chip. Figure 2 below shows the components of a microcontroller system.

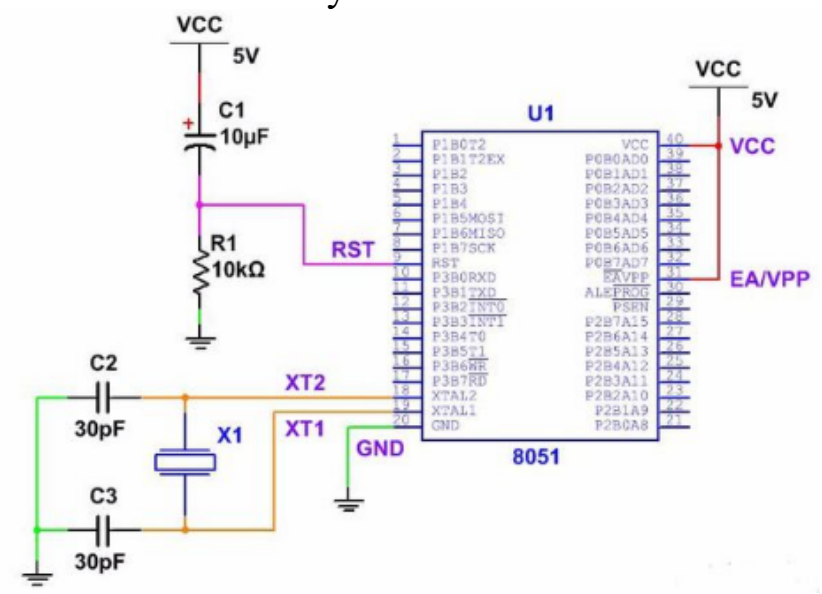

\subsection{Digital Tube Display}

Figure 2. Components of the SCM System

The digital tube, also called glow tube, is an electronic device that can display numbers and other information. The glass tube includes an anode made of a wire mesh and a plurality of cathodes. Most digital tube cathodes have a digital shape. The tube is filled with low pressure gas, usually mostly helium plus some mercury and/or argon. To charge a certain cathode, the digital tube will emit color light, depending on the gas inside the tube, generally orange or green. Although similar in appearance to the vacuum tube, the principle is not to heat the cathode to emit electrons. It is therefore called a 
cold cathode tube or a variant of neon light. At room temperature, the temperature of such tubes rarely exceeds $40^{\circ} \mathrm{C}$ even under extreme indoor operating conditions.

The most common form of nixie tube is 10 cathodes. The shape is the numbers 0 to 9 , and some nixie tubes have one or two decimal points. However, there are other types of digital tubes that display letters, symbols, and symbols. Like a "digital tube", its cathode is a mask made of a template with a numerically shaped hole. Some Russian digital devices, such as IN-14, use the inverted number 2 to represent 5, presumably to save production costs, without obvious technical or aesthetic reasons. Most of Russia's digital tubes use inverted 2 as 5.

A DC voltage of 170 volts is applied between the cathode and the anode, and each cathode emits bright red-orange light. Due to the different gas mixtures, different types of digital tubes have different colors. Longevity nixie tubes incorporate mercury in their manufacture, reducing sputtering, resulting in a blue or purple tone. In some cases, these colors are filtered by a red or orange filter coating on the glass. Figure 3 below is a common digital display.

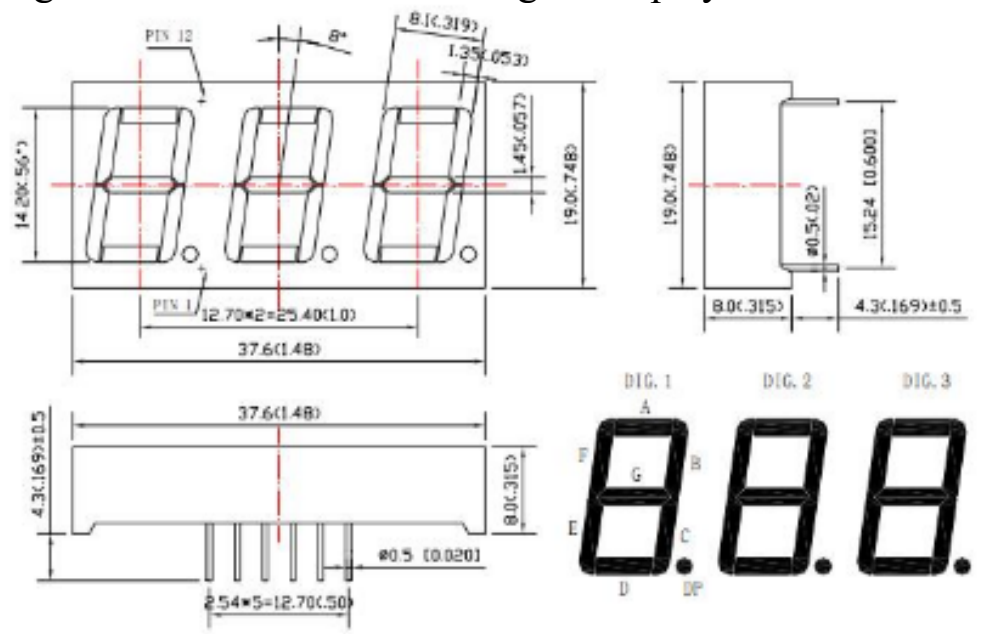

\subsection{Alarm Circuit}

Figure 3. Digital Tube Display

Alarm circuit A circuit that reports the machine status or dangerous conditions to the operator in a visual display or an audible alarm. In the design of the car reversing her car, when the distance from the vehicle's tail reaches a certain range, the alarm circuit will be connected and an alarm sound will be issued. Figure 4 below shows a schematic of the alarm circuit.

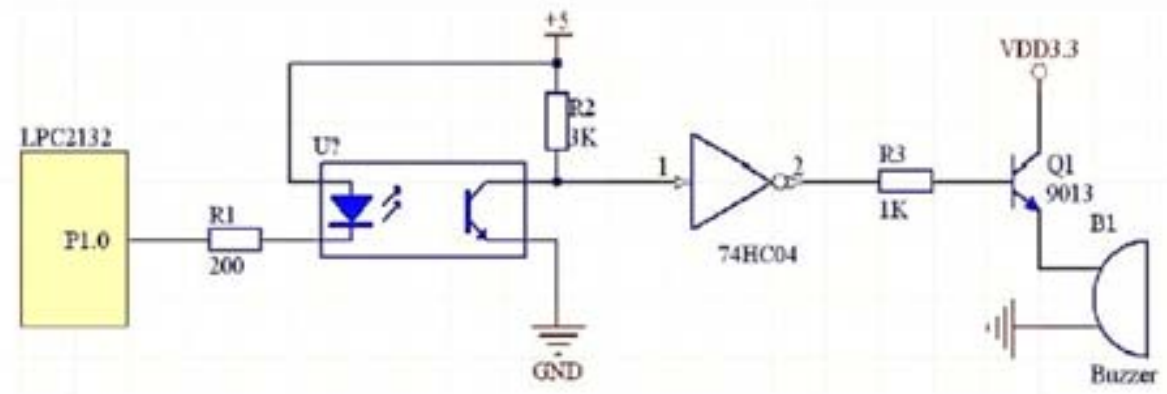

Figure 4. Alarm circuit

\section{Hardware Design of Vehicle Reversing System}

The design of hardware circuit mainly includes single-chip system and display circuit, ultrasonic transmission circuit and ultrasonic detection and reception road. The microcontroller uses STC89C51 or its compatible series. A $12 \mathrm{MHz}$ high-accuracy crystal oscillator is used to obtain a stable clock frequency and reduce measurement errors. The microcontroller uses a P2.4 port to output the $40 \mathrm{kHz}$ square wave signal required by the ultrasonic transducer, and uses the external interrupt 0 port to monitor the return signal output by the ultrasonic receiving circuit. 
Display circuit: The current measured distance can be displayed by four digital tubes, and can be accurate to millimeters. In order to save the precious IO resources of the microcontroller, the display circuit shares the P0 port, so the latch is used to latch the digital pipe segment selection and the bit selection data. The latch selects the 74HC573. Because the microcontroller P0 port drive current is small, so it is necessary to use Pull the resistor to increase its drive capability. The display circuit is shown in Figure 5 below:

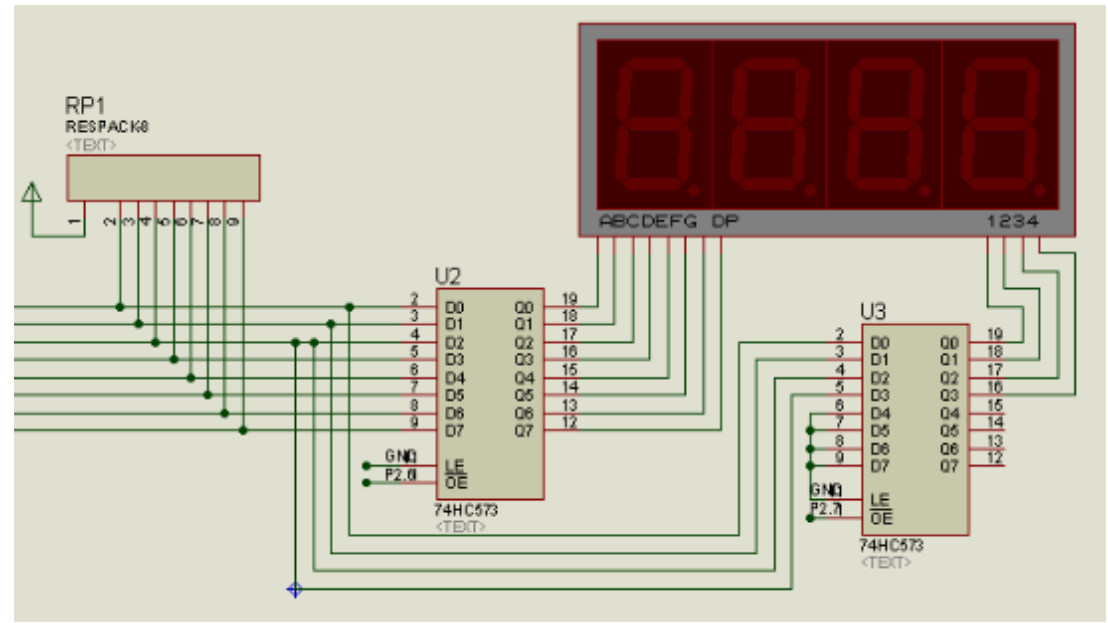

Figure 5. Shows the Circuit

\section{Software Circuit of Car Reversing System}

The system software design adopts a modular design, including main program design, T0 interrupt service routines, external interrupt service routines, distance calculation subroutines, and digital tube display subroutine design. The program has two measurement modes to choose from, namely automatic measurement and manual measurement mode. After the system is started, the mode selection is first performed, long press to enter automatic measurement, and short press to enter manual measurement. This kind of scheme is more humane and easier to operate.

The main program is first initialized to the system environment, set timer 0 to count, set timer 1 timing. Set the total interrupt enable bit EA. After the program main program is executed, the timing ranging judgment is performed. When the distance measurement flag ec $=1$, the measurement is performed once. In the program design, the ultrasonic ranging frequency is 4-5 times per second. In the ranging interval, the entire program mainly displays the measurement results in a loop. When the ultrasonic ranging subroutine is invoked, four ultrasonic pulses with a frequency of $38.46 \mathrm{kHz}$ are first generated by the single-chip microcomputer and loaded on the ultrasonic transmission head. After the ultrasonic head sends the ultrasonic wave, it immediately starts the internal timer T0 for timing. In order to avoid the direct wave trigger caused by the ultrasonic wave transmitted directly from the transmitting head to the receiving head, the single-chip microcomputer needs a time delay of about 1.5 -2 ms (this is The ultrasonic distance meter will have a minimum measurable distance, called the blind spot value, before starting the level determination procedure for the P3.5 pin of the microcontroller. When the level of P3.5 pin is detected to go from high to low, T0 timing is stopped immediately.

When designing a subroutine for generating an ultrasonic wave, in order to facilitate program shifting and accurate generation of an ultrasonic signal, the ultrasonic generating program of this range is an advanced sound wave generating program written in assembly language.

\section{Experimental Debugging Results}

After the circuit and the components are determined, the circuit is first laid out. The method adopted is a block soldering circuit, that is, the ultrasonic module is decomposed into a transmitting portion and a receiving portion. After the welding is completed, download the program to the SCM for debugging. The following problems are found: 1 . The digital tube does not display the distance. 
After the debugging, it is found that the program cannot enter the interrupt program. 2. After the modification, the digital tube can display the distance, but it can only be accurate. To centimeters. The reason is that there is a problem with the program structure, that is, the timer is not turned on immediately after the ultrasonic wave is transmitted. 3. After the program adds two measurement modes, manual and automatic, the measurement mode is selected by pressing the key. The system does not give a prompt and it is not convenient to operate.

The corresponding solutions are as follows: 1. Modify the debugger program so that the program can enter the interrupt normally; 2. Re-adjust the program structure to make it more streamlined; 3. Add the light-emitting diodes to the hardware and add the corresponding code to the program so that the user can select the mode. The system can give tips and be more human.

\section{Conclusion}

Today's electronic products in the world are not simply hardware components. Most of them are made up of hardware and software. The software is responsible for the operation of the system. The hardware is the basis of the circuit. Just like the car radar system designed this time, the hardware circuit must have functions such as ultrasonic transceiver, display, and alarm, and the software links these modules to form a complete system. Therefore, when designing a system, even if it is a simple system, we should proceed from the entire design, decompose it into functional modules, and finally combine these functional modules to form a complete system. This simplifies the design of the system.

\section{References}

[1]. Xu Wei. Design and application of reversing radar system based on S3C44BOX [D]; Jilin University; 2006.

[2]. Bi Ran. Design and development of reversing radar based on embedded system [D]; Jilin University; 2009.

[3]. Liu Shuxin Cao Yundong Hou Chunguang Liu Xiaoming. Application of Embedded System in Circuit Breaker Intelligent Control [A]; [C]; 2007.

[4]. Yan Chenggong, Lan Zhaohui, Chen Shuo. Ultrasonic Ranging System Based on Single Chip Microcomputer [J]; Application Science \& Technology; 2004-07. 